\title{
Survey on the Use of the Mobile Learning Based on Mobile Cloud Computing
}

http://dx.doi.org/10.3991/ijim.v10i3.5672

\author{
Z. Rimale, E. Benlahmar, A. Tragha and K. El Guemmat \\ Faculté des Sciences Ben M'Sik, Casablanca. Morocco
}

\begin{abstract}
- with the continued growth of wireless technology and the development of mobile computing, mobile learning (m-learning) has become one of the most important ways of learning. However, the traditional way of m-learning has a lot of limitations and disadvantages. For some time the Mobile Cloud Computing (MCC) is an adoptable technology for most organizations with dynamic scalability and utilization of virtualized resources as a service over the Internet. MCC is growing rapidly, with applications in almost all areas, including education. Today, there are several researches confirming that $\mathrm{MCC}$ is a good way to overcome the disadvantages and limitations associated with the traditional way of $m$-Learning. This article is an attempt to clarify the definition and benefits of m-learning and MCC while presenting their existing work.
\end{abstract}

Index Terms-Key words: Mobile Cloud Computing, mLearning, d-Learning, e-Learning, XML.

\section{INTRODUCTION}

Recent years have seen the rise of m-Learning, favored by the continuous development of mobile computing and wireless technology [1]. The M-Learning can be defined as a subdomain of E-learning in which applications include mobility. In the M-learning, the learner can be mobile, i.e. it is not required to remain physically in the classroom. One of the main objectives in an m-Learning environment is to provide learners with the right resource at the right time and in the best way.

With the growing number of users, services, learning content and resources, in addition to the limits of mLearning environments that are related to mobile devices used by learners such as reduced processing power, battery and the limited size of the screen [2]. To this end, how to deploy m-learning applications becomes problematic.

To improve the quality of traditional m-learning and achieve its objectives, it was suggested a promising technology including the MCC that overcomes the limitations and disadvantages associated with this mode of learning.

Recently, MCC is considered computer model of offering IT services in the form of on-demand services, accessible from anywhere, anytime and by anyone. MCC offers guaranteed dynamic IT environments reliable, customized and quality of service for end users. MCC has many advantages in education as resources stored in the cloud are accessed by the student and the teacher via mobile devices [3].

M-learning has become an important topic for researchers in computer science and education. There are many works that have implemented an m-learning environment taking into account various problems related to mobile devices such as bandwidth, battery, context, content and adaptation, etc.

This article attempts to clarify the definition of $\mathrm{m}$ learning in a MCC environment is a practical way to overcome its limitations and disadvantages. This paper is organized as follows: Section II identifies areas of IT-related m-learning (e-learning, distance learning), its advantages and disadvantages. Section III describes the MCC, its services, deployment models, architecture and some advantages in using the m-learning. Section IV describes some traditional m-learning work. Section VI describes some work on m-learning based on the MCC. Section V1 ends this book with the conclusion and future work.

\section{E-LEARNING AND M-LEARNING}

\section{A. Context}

E-learning is a distance learning mode based on new multimedia technologies and the Internet, which allows one or more persons to form from their computer. Multimedia materials used can combine text, graphics, sound, image, animation and even video [4].

Many researchers consider the m-learning just as a natural evolution of e-learning, which completes a missing piece of the solution while leveraging the main strength of mobile ICT, or as a new stage of distance learning (dlearning) and e-learning [5].

Historically, the d-learning has over a hundred years of experience and tradition. Its main feature is the separation in time and space, the learner and the teacher. The eLearning provides new distance learning methods based on information technology and the Internet. The other side of the m-learning is part of the e-learning and, therefore, a portion of d-learning as shown in Fig 1[5].

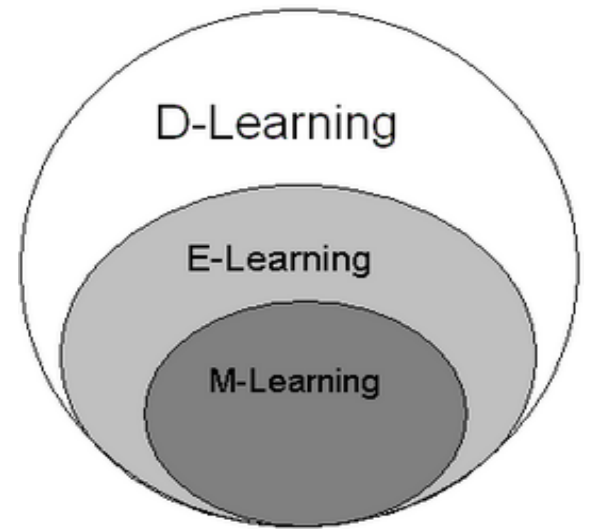

Figure 1. The positioning of $\mathrm{m}$-Learning as part of the e-Learning and d-Learning [5] 
One definition can summarize what is usually understood by the term m-learning is: "Any sort of learning that happens when the learner is not at a predetermined location, fixed, or learning that happens when the learner takes advantage of the opportunities offered by mobile technologies "[6].

\section{B. Advantages of the M-Learning}

In view of large group of users of mobile devices, Sharples, 2003 [7] considers that educators must find ways to exploit the potential of mobile technologies to allow them to benefit from their advantages in learning:

- Learners can interact with each other and with the practitioner instead of hiding behind big screens.

- It is much easier to accommodate several mobile devices in a classroom than several desktop computers.

- PDAs, Tablet PCs are lighter and less bulky than bags full of files, paper and textbooks, or even laptops.

- Writing with the stylus is more intuitive than using the keyboard and mouse.

- Mobile devices can be used anywhere, anytime.

- These devices engage learners; young people have lost interest in education, such as mobile phones, gadgets and gaming devices.

- The size of these devices is smaller and lighter

- Mobile devices have lower prices [5].

According to "EDUCAUSE" M-Learning benefits are many [2]:

- Extending the teaching and learning in spaces beyond the classroom,

- Provide teachers and learners increased flexibility and new opportunities for interaction,

- Support collaborative learning experiences, accessible, integrated into the world beyond the classroom,

- Facilitating learning on the workplace: people can continue their work while forming (thus increasing efficiency in the workplace and therefore productivity)

- Facilitate access to training in rural or remote areas

- Low cost, portability.

\section{Disadvantages of M-Learning}

Despite the advantages provided by the use of mobile devices in learning, they have potential limitations that may be [2]:

- The readability of the screens of mobile devices (small screens of mobile and PDA) which limits the amount and type of information that can be displayed.

- The limited memory size, the latest phones have the ability to expand the memory size by adding memory cards.

- The autonomy of the devices, the batteries must be recharged regularly, and data can be lost if it is not done properly.

- They can be much less robust than desktop computers (although tablet PCs begin to address this issue).

- It is difficult to use animated graphics, especially with mobile phones, although $3 \mathrm{G}$ and $4 \mathrm{G}$ will allow.
- The data rate, but this is much improved with $3 \mathrm{G}$.

- The hardware obsolescence of speed, this is a rapidly changing market, especially for mobile phones, devices can become obsolete very quickly.

- Bandwidth may degrade with a larger number of users when using wireless networks.

\section{MoBile Cloud COMPUTING}

\section{A. Context}

The Mobile Cloud Computing Forum defines MCC as follows [3]: « Mobile Cloud Computing refers to an infrastructure where storage and execution of data products to external mobile devices. Mobile applications in the cloud will move the power and data storage away from mobile devices to the cloud»

The MCC expression was introduced after the concept of «Cloud Computing » which was launched in mid-2007. It has been attracting the attention of entrepreneurs as a profitable business option that reduces the cost of development and operation of mobile applications and mobile users as a new technology to achieve a rich experience of a variety of mobile services at low cost, and researchers as a promising solution for green iT core [3].

\section{B. Service Models:}

Cloud services, illustrated in Figure 2, are generally based on three layers [3]:

- Data center layer provides the necessary equipment and infrastructure clouds. In this layer, there are a number of servers connected to broadband networks. Data centers are often located in places with the ability to power high voltage and away from all dangers.

- IaaS (Infrastructure as a Service) form the base of the cloud. These servers, storage, databases and network equipment leased as demand services with levels of adjustable services.

- PaaS (Platform as a Service) is a runtime platform hosted by an operator connected to the Internet. It allows you to run software in SaaS mode. For years, Amazon, Google and others offer PaaS solutions at competitive prices.

- SaaS (Software as a Service) is an application provided as a service. Users access it via a connection to the Internet. Updates are regularly made by the publisher of the software and all customers benefit immediately.

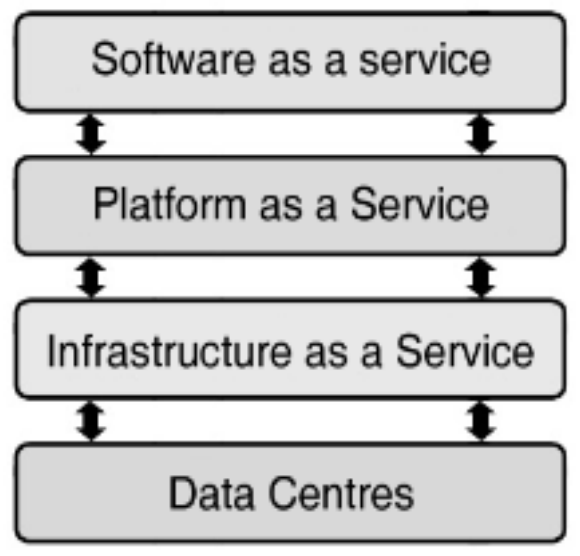

Figure 2. Architecture of Service-Oriented Cloud Computing [3] 
C. Deployment Models:

There are three forms of deployment of Mobile Cloud Computing [8]:

- Private Cloud: If the cloud infrastructure is used by a single company, the cloud is private said.

- Public Cloud: In case some large companies can exploit large through internet data centers, making them accessible to everyone. The most reputable companies offering this service are Google, Amazon, and Microsoft.

- Hybrid Cloud: A hybrid cloud is a case of combination of at least two types of Cloud above.

\section{Architecture of Mobile Cloud Computing:}

The general architecture of MCC can be shown in Figure 3 [3]. Mobile devices are connected to mobile networks via base stations (BTS, access point, or satellite) that establish and control the connections and functional interfaces between networks and mobile devices. Applications and mobile user information is transmitted to the central processors that are connected to servers providing mobile network services. Here, mobile operators can provide services to mobile users as AAA (Authentication, Authorization, Accounting) based on the home agent (Home Agent: HA) and data stored in the subscriber database. After that, requests the subscribers are delivered in the cloud through the Internet.

In the cloud, controllers handle applications to provide mobile users with the corresponding cloud computing services. These services are developed with the concept of utility computing, virtualization and service-oriented architecture.

\section{Benefits Of Using The M-LEARNING BASED Mobile Cloud COMPUTING}

M-learning can use and benefit from mobile cloud computing using:

- IaaS: use a solution of m-learning on the provider's infrastructure.

- PaaS: use and develop a solution of m-learning based on the supplier's development interface.

- SaaS: Using m-learning solution provided by the provider.

There are many benefits when the m-learning is implemented with MCC technology, namely [13]:

- Low cost: The m-learning users should not have cell phones upscale with a good configuration to run the m-learning applications. They can run cloud applications through their mobile phones with a minimum configuration with Internet connectivity.

- Improved performance: Since m-learning applications based on the MCC run on the cloud, mobile client laptops perform better because they have less memory loaded programs and processes

- Updates instant software: Because applications are in the cloud, updates occur automatically and are available once learners are connected to the cloud.

- Improved document format compatibility: In the MCC, we have more compatibility to open files and applications easily without installing more software on mobile devices seen as m-learning based on the MCC opens files via the cloud.

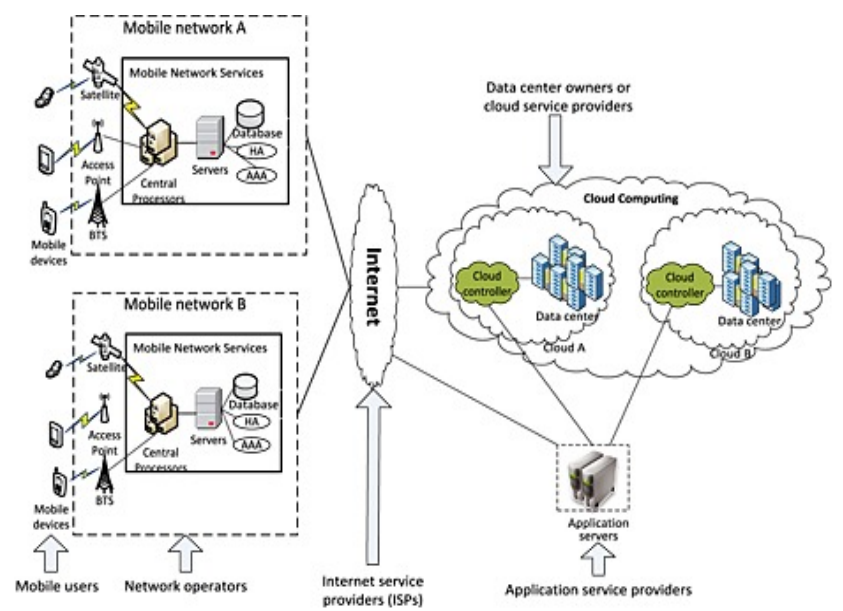

Figure 3. Mobile Cloud Computing (MCC), architecture [3]



Figure 4. general and generic architecture of m-learning [9]

- Benefits for students: Students get more benefits through the m-learning based on the MCC. They can take online courses, participate in online examinations, obtain feedback on the course instructors, and send their online projects to their teachers.

- Benefits for teachers: Teachers also get many benefits via m-learning based on the MCC. Teachers are able to prepare for online tests for students, process and create better content resources for students through content management, evaluate the tests, homework, projects, followed by students, post comments and communicate with students through online forums.

- Data security: A great concern is the safety of data because the software and data resides on remote servers that can block or disappear without additional warnings. While this does not seem very reasonable, mobile cloud computing provides significant security benefits for individuals and companies that use / develop m-Learning solutions.

\section{M-LEARNING WORKS}

\section{A. A general architecture developed on the platform of e-learning}

Since the m-learning is considered an extension of elearning, most of researchers have proposed architectures developed on e-learning platforms, while adding features to the platform to adapt to mobile devices [9]. M-learning can be used on platforms of e-learning while adding new features mobile technology as shown in the figure 4 . 
This architecture considers the m-learning as an extension of an LMS (Learning Management System) Traditional and provide services tailored to mobile users .It is composed of two layers, one layer e-LMS (e-Learning Management System) and layer m-LMS (Mobile Learning Management System).

In traditional e-LMS layer, the features can be grouped into four categories i.e. learning resources, specific learning services, common services and presentation. In the $\mathrm{m}-$ LMS layer, three blocks are added on top of the e-LMS layer including the discovery of context, packaging and synchronization, management of mobile content and presentation of adaptation. It is an architecture that consists of two layers allows the flexibility and scalability to support the various learning activities. This architecture is likely to be supplemented and developed for a specific situation according to the needs and constraints of each.

\section{B. Approach based on web services}

Sushi et al. (2004) [10] propose an architecture of web services for m-Learning; it provides students and teachers with the opportunity to obtain all of the learning material related to their handheld devices through a Web services architecture. This architecture consists of four layers: the application layer, integration using web services standards, delivery devices and human layer. This architecture is shown in Figure 5.

- Application layer: This layer consists of various services for students and instructors. These services are created by administrators and instructors for use by students. The other layers below the application layer will be completely transparent to the students.

- The integration layer using web services standards: This layer includes all the content and applications that may already be available in different formats. The Web services architecture used for such purpose would make the entire integration process like ready to use, and provide enough flexibility to allow independent content features.

- The layer of the delivery devices: This layer is used to deliver the content using the Internet and allowed multiple devices.

- The human layer: This layer consists of students, administrators, instructors.

\section{Adaptive Approach and Context-Aware for m- \\ Learning}

In the context of m-Learning, it is helpful to consider the context sensitivity and adaptability as two sides of the same coin. The goal of adaptability and sensitivity to context is to better support a variety of learners they may have very different skills and motivation to learn in varying contexts [11].

\section{1) Adaptation of learning}

Research in the field of adaptation of learning following three directions [11]:

- Adaptation of content and course: It consists in adapting the course to suit the preferences of the learner, their previous knowledge, purpose, and deliver appropriate courses to the characteristics and capabilities of mobile devices learners.

- The presentation of adaptation: The content was presented in different ways based on learners' prior competences (knowledge and skill prerequisites) and learner preferences.

- Adaptation of navigation and sequencing: It adapts the navigation and rating links to the user, to help him find a suitable path in the learning process.

\section{2) Sensitivity to context (context-awareness)}

Much has been written about the context, and several researchers have tried to put forward a definition according to context their views. The most cited definition is that of Dey (2000) [12] which define the context as any information that can be used to characterize the situation of an entity. The entity is a person, a place, an object that is considered relevant to the interaction between the user and the application, including the user and the application itself.

Among the contextual learning systems or context sensitive learning we chose a representative example of this type of systems. A common feature of context aware systems is to have a contextualization module to adapt the learning activity and content to user contexts.

The above system is a typical architecture of contextual systems. It includes a LCMS (Learning Content Management System), which is developed on technologies of elearning. The content stored in the system corresponds to the standards of e-learning, described by XML files. To

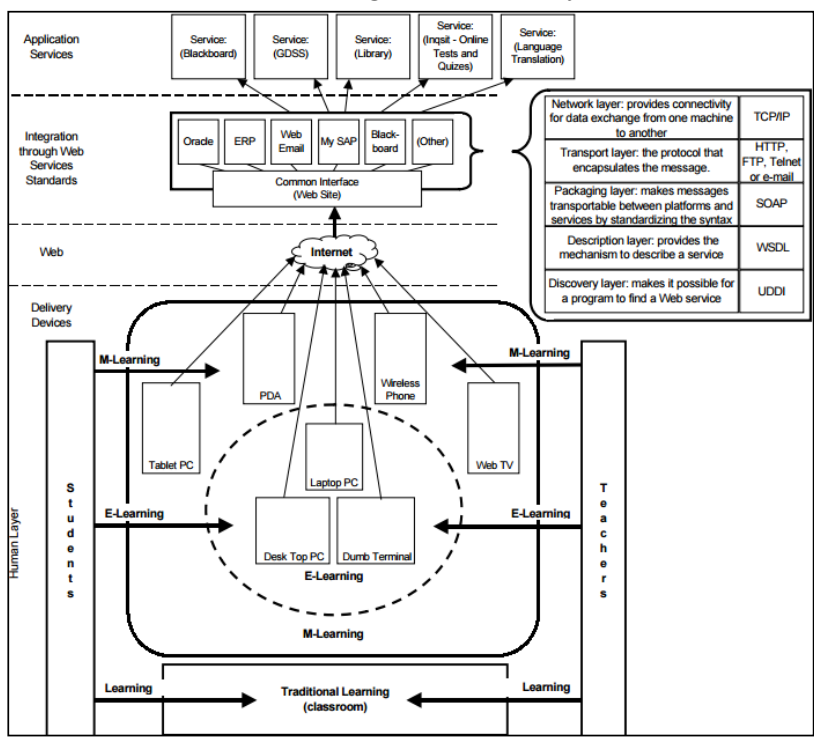

Figure 5. flexible architecture services for m-Learning [10]

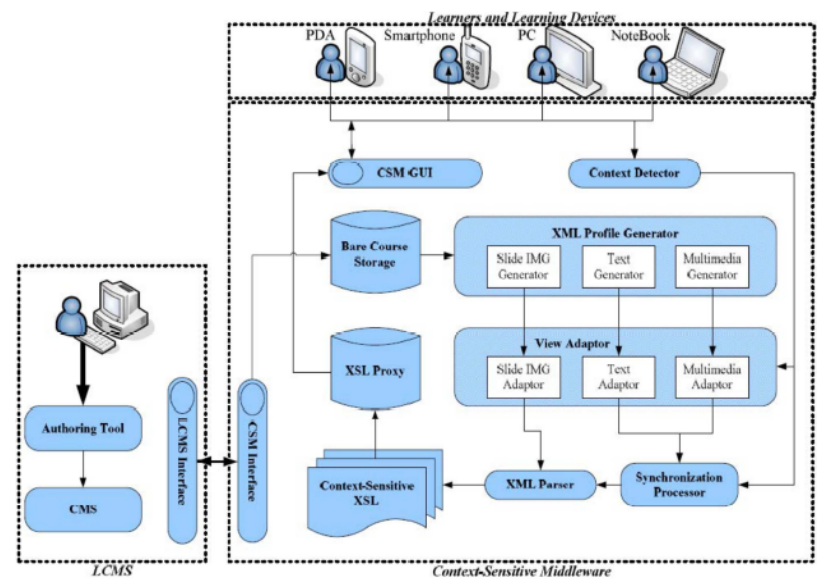

Figure 6. Architecture context sensitive system [13] 
adapt the content to the mobile context, a CSM module (Context-Sensitive Middleware) is added between the LCMS and mobile devices.

\section{Approaches based agents}

Agent technology has been used by many m-Learning systems, some of them [14] [15] have used the intelligent agents to adjust the learning content dynamically throughout the learning process.

\section{1) Platform based agent (Bee-gent framework)}

Bee-gent platform is used to implement mobile agents in m-Learning system to improve its environments and solve its shortcomings. Bee-gent is composed of two types of agents: wrapper agent and mediation officer [14]. Figure 2-6 shows how Bee-gent is used to implement mobile agents in the system. The scenarios of main process are.

a) On the server side, the inference engine interacts with the learner model and the module of experts through the database interface.

b) On the client side, if a request should be sent to the server, the client informs the client side agent wrapper, and it will create a mediation officer who is able to carry the information to the server.

2) Approach Mobile Agents in m-Learning Environment

Sheng-Hung and Chung [15] propose an approach that uses mobile agents and ACID (atomicity, consistency, isolation and durability) in the m-learning. The above approach uses two agents; Replication Agent and Snapshot Agent that are proposed as an effective way to solve the limitations of m-learning namely the heavy load and bandwidth limit. The implementation of mobile agents, such middleware for m-Learning environment is to provide database replication between learners and offsite databases.

In this technique proposed in Figure II-8, a central database LMS is determined as a Snapshot publisher, where a compact edition of files and information is generated Snapshot rental residing on the database server Database. Users can quickly retrieve information from wireless mobile applications. In this case, the Replication Agent provides the flexibility to move from one site (user) to another (central database) for the synchronization of critical data.

The replication agent has the following components: publisher, distributor, Subscribers, publications, articles, and subscriptions. Replication agents to be used in the environmental field of mobile applications that are responsible for copying and moving large data fields between the publisher and subscribers are: Snapshot Agent, Log Reader Agent, Distribution Agent, queue Reader Agent, and Merge Agent. The following figure shows the replication process.

\section{M-LEARNING WORKS ON MCC}

\section{A. A m-Learning architecture based on the Cloud}

The main objective of M-Learning in the cloud environment is to provide learners with the resources centralized shared knowledge anytime and anywhere. An architecture that has been proposed for m-learning as shown in Figure 1 includes the communication between the end user device (terminal) and the data center into a cloud environment [16]. The terminals can be connected to the infrastructure within the local network of the University (LAN: Local Area Network), or they can be connected to external networks (Internet). The platform of the university server (Course Management System) [17] hosts educational resources via the university LAN. A user can access the platform directly from the university LAN or across the Internet to gather learning materials.

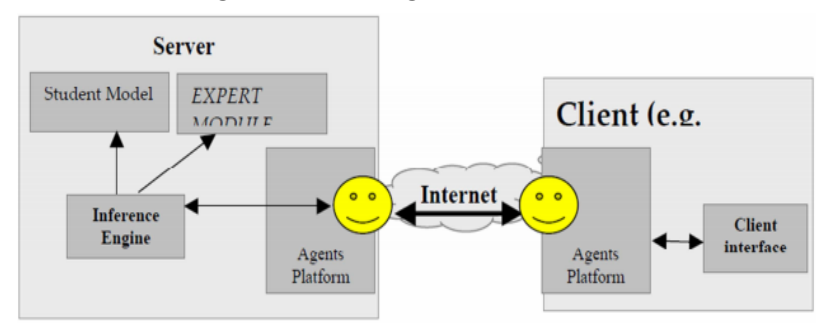

Figure 7. Architecture of m-learning environment using mobile agents [14]

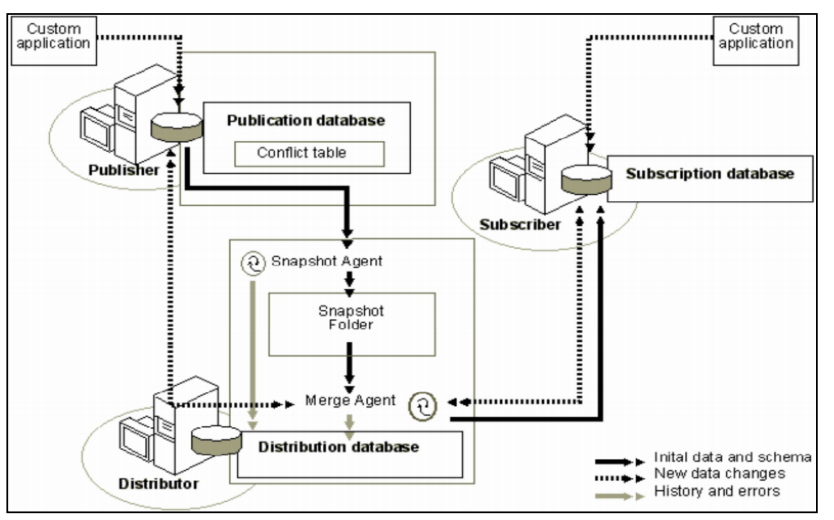

Figure 8. Replication process [15]

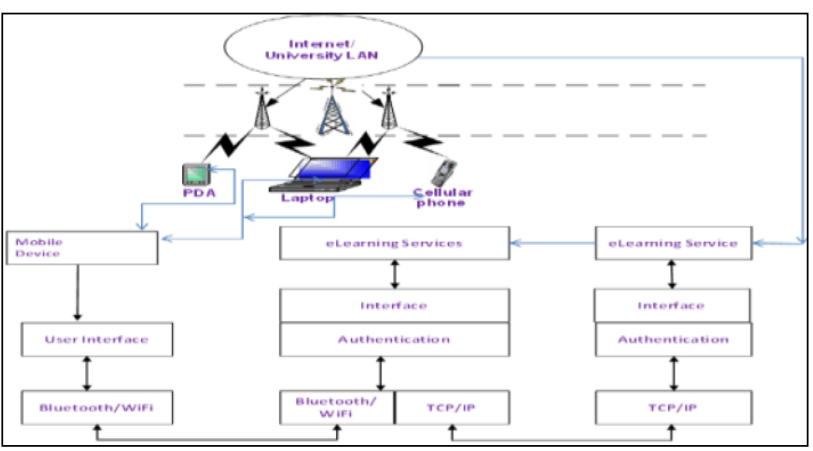

Figure 9. Architecture of mobile cloud learning [16].

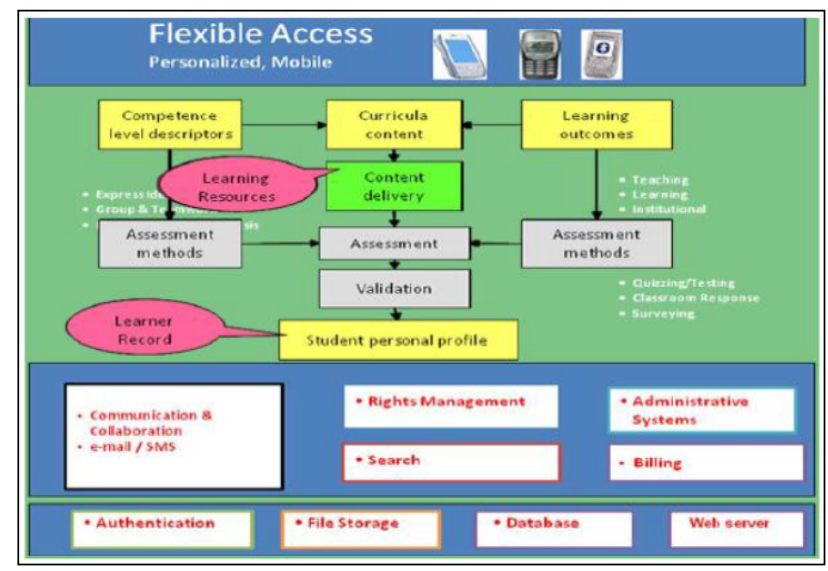

Figure 10. Details Of Architecture Components. 
The user can access the data center, LAN or from the university, or directly from the Internet. The authentication server manages authorized access to the data center, and is connected directly to the assets and liabilities servers as shown in Figure 9.

\section{B. eLSaaS: A Framework for adapting the content of m- learning}

This section provides a solution for the construction of a virtual and personalized learning environment that uses cloud technology to create a model based on services for suppliers and m-learning learners. The concept of eLSaaS presented as a software distribution model in which applications are hosted by a service provider and distributed via the Web.

In the proposed environment Elsass as shown in Figure [11], it is easy to combine semi-structured data, stored in a native XML database (NXDB) with structured data stored in a relational database (RDB) through the Web services (WS). The objective is to provide direct data and application integration, located on sites distributed to improve the achievement of learning outcomes. This approach promotes independent m-learning gateway device between different mobile units and the huge number of learning resources available on a plethora of LMS (Learning Management System).

It becomes possible by combining the Open Semantic Editor Web-based [19] with a set of additional services to accommodate different mobile units with direct access to learning objects (Los) usually designed for desktop Web browsers. A semantic content adaptation service is connected to the normalization of the content. This tool uses templates to automatically and efficiently tailor content for mobile web browsers.

An alternative service is available for a voice solution, which allows learners to turn any written text in the files of the natural voice, when using the standard voice. This approach enables both the generation and progressive download text and audio based on the dynamic learning materials for m-learning and ubiquitous access.

\section{CONCLUSION AND FUTURE WORK}

The m-Learning based on the MCC positively affects the learning process. Students and teachers have the opportunity to quickly and economically to different platforms and application resources through mobile phones on demand. These automatically reduces the cost of organizational costs while delivering more powerful functional capabilities and gradually eliminate the costs of software licenses, cost of materials and cost of maintenance.

Our future research aims to define and develop a semantic web tool for the description of a learning object structure using XML scheme based on an anthology to facilitate the creation and sharing of these items with the content XML via the Mobile Cloud Computing environment to overcome the limitations and disadvantages of the traditional method of m-learning.

\section{REFERENCES}

[1] Zouhair Rimale, El Habib Ben Lahmar, Abderrahim Tragha, A Brief Survey and Comparison of m-Learning and e-Learning, International Journal of Computer Networks and Communications. Vol.4, Issue 4, April 2016

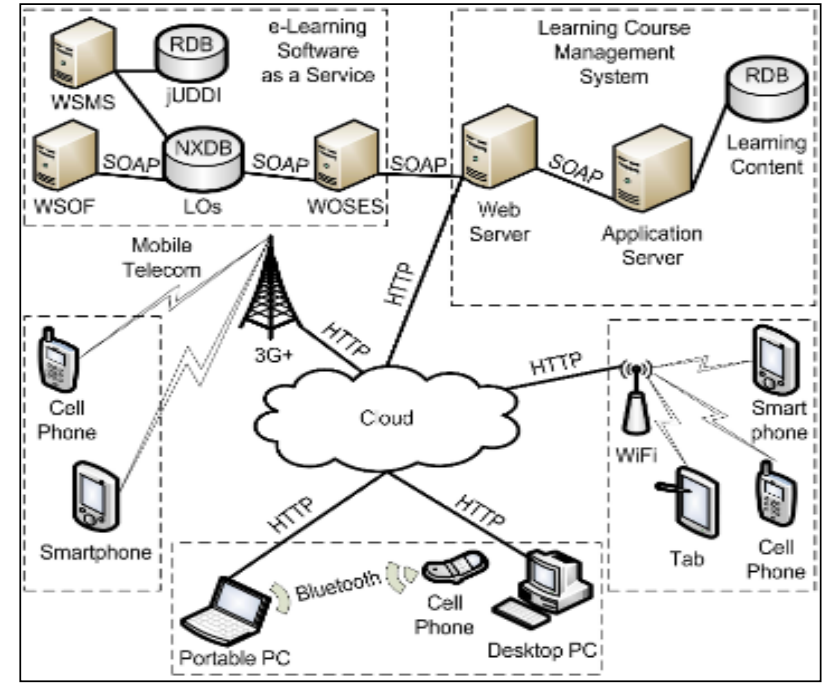

Figure 11. e-Learning software as a service solution (eLSaaS) [18]

[2] Nane Yaw Asabere, Benefits and Challenges of Mobile Learning Implementation: Story of Developing Nations, International Journal of Computer Applications (0975 - 8887) Volume 73- No.1, July 2013

[3] H. T. Dinh, C. Lee, D. Niyato and P. Wang, "A survey of mobile cloud computing: architecture, applications, and approaches", Wireless Communications and Mobile Computing - Wiley, (2011) October

[4] Joi L. Moore, Camille Dickson-Deane, Krista Galyen "e-Learning, online learning, and distance learning environments: Are they the same?" internet and Higher Education 14 (2011) 129-135 http://dx.doi.org/10.1016/j.iheduc.2010.10.001

[5] Georgiev, S, Georgieva, E, Smrikarov, A, "M-Learning - A New Stage Of ELearning", Proceedings Of The 5th Intern. Conference On Computer Systems And Technologies - Compsys Tech'2004, Rousse, Bulgaria, Pp. Iv.28-1-5, 2004.

[6] O'Malley, C. et al. 2003. Guidelines for learning/teaching/tutoring in a mobile environment. Mobilearn project deliverable. http://www.mobilearn.org/download/results/guidelines.pdf

[7] Sharlples M. Disruptive Devices: Mobile Technology for Conversational Learning. International Journal of Continuing Engineering Education and Lifelong Learning. 2003;12(5/6): Pp. 504-520. 2003.

[8] Pragaladan. R, Leelavathi .M, A Study of Mobile Cloud Computing And Challenges. International Journal of Advanced Research in Computer and Communication Engineering Vol. 3, Issue 7, July 2014

[9] Anna Trifonova, Marco Ronchetti, A general architecture to support mobility in learning. Proceedings of the IEEE International Conference on Advanced Learning Technologies (ICALT'04) 0-7695-2181-9/04 \$20.00 (C) 2004 IEEE http://dx.doi.org/10.1109/ icalt. 2004.1357368

[10] Sushil K. et al., Web Services Architecture for M-Learning, Electronic Journal on e- Learning Volume 2 Issue 1, USA, Pp. 203216, February 2004.

[11] Anastasios A. Economides, Adaptive context-aware pervasive and ubiquitous learning. Economides, A. A. (2009). Adaptive contextaware pervasive and ubiquitous learning. International Journal of Technology Enhanced Learning, Vol. 1, No 3, pp. 169-192, Inderscience. ISSN (Online): 1753-5263, ISSN (Print): 1753-5255.

[12] Dey, A.K. and Abowd, G. 'Towards a better understanding of context and contextawareness', Proceedings 2000 Conference on Human Factors in Computing Systems (CHI), The Hague, The Netherlands. 2000.

[13] C.Chu W., et al. Context-Sensitive Content Representation for Mobile Learning. ICWL2005- the 4th International Conference of Web-Based learning. Hong Kong, China; 2005.

[14] Kinshuk, Taiyu Lin, Improving mobile learning environments by applying mobile agents technology, Massey University, Palmerston North, New Zealand, 2003. 
[15] Sheng-Hung, Chung, Mobile Agents Approach in Mobile Learning Environment - ACID Mobile Transaction, ICI9 - International Conference on Information; Kuala Lumpur, Malaysia,12 - $13 \mathrm{Au}-$ gust 2009.

[16] Abu Taha Zamani The Impact of the Cloud Based M-Learning in Higher Education, International Journal of Advanced Research in Computer Science and Software Engineering,Volume 4, Issue 1, January 2014

[17] Hossain Masud, X. Huang, ?An E-learning System Architecture based on Cloud Computing?,World Academy of Science, Engineering and Technology, Vol: 62, 2012.

[18] Ivan Madjarov, Omar Boucelma eLSaaS: A Framework for Mobile Learning Content Adaptation. CSEDU, 2012

[19] Ivan Madjarov, Omar Boucelma, 2010. Learning Content Adaptation for m-Learning Systems: a Multimodality Approach, The 9th ICWL, LNCS 6483, pp. 190-199.

\section{AUTHORS}

Zouhair Rimale is a PhD candidate at the Faculty of Sciences Ben M'Sik, Casablanca, Morocco, his research fields include m-learning, cloud computing, educational modeling, semantic indexing. He's Microsoft Certified
Technology Specialist (SharePoint, ASP.NET, and HTML5). He works as a Microsoft consultant.

Benlahmar El Habib is a professor of higher education at the Faculty of Sciences Ben M'Sik, Casablanca, Morocco. His Research interests include metasearch, information retrieval, semantic web, mobile, data science, automatic processing of natural language.

Abderrahim Tragha is a professor of higher education and Research Director, Laboratory Information Technology and Modeling at the Faculty of Sciences Ben M'Sik, Casablanca, Morocco. His Research interests Theory of Computation, Computer Security and Reliability, Artificial Intelligence.

Kamal El Guemmat is a $\mathrm{PhD}$ in computer sciences, Research fields: Semantic Indexing, Semantic Web, Information Retrieval Systems, Automatic Processing of Natural Language, Information and communication technology for teaching and learning, Educational modeling, E-Learning.

Submitted 22 March 2016. Published as resubmitted by the authors 16 May 2016. 